\title{
Estimate a Flexible Link's Shape by the Use of Strain Gauge Sensors
}

\author{
M. H. Korayem, A. M. Shafei, and F. Absalan \\ Mechanical Engineering Department, Iran University of Science and Technology, Narmak, Tehran 13114-16846, Iran \\ Correspondence should be addressed to A. M. Shafei; shafei@iust.ac.ir
}

Received 29 June 2012; Accepted 8 August 2012

Academic Editors: D. K. Pratihar and A. Sabanovic

Copyright (c) 2013 M. H. Korayem et al. This is an open access article distributed under the Creative Commons Attribution License, which permits unrestricted use, distribution, and reproduction in any medium, provided the original work is properly cited.

\begin{abstract}
This paper presents a method for estimating the flexible link's shape by finite number of sensors. The position and orientation of flexible link are expressed as a function of curvature of the link. An interpolation technique gives this continuous curvature function from a finite set of the Wheatstone bridge made with strain gauges. For interpolation we can use different functions to find better way for estimation of link's shape. Comparison between different types of function can show us best corresponding with nature of the link. Our case study is a single flexible link robot. A high-precision data logger is used as data acquisition instrument.
\end{abstract}

\section{Introduction}

Research into flexible manipulator fields has become more important for more than a decade on account of new robotic applications in different areas such as industrial tasks in which the trend is to use lightweight materials (e.g., carbon fiber and composites) in the structures of manipulators in order to improve the performance of the industrial robots which are large and heavy. In aerospace applications the lightness of the materials is also an important requirement; the control of large structures, such as boom cranes and fire rescue turntable ladders, which are treated as flexible link robots is discussed by $[1,2]$; minimally invasive surgery is performed with thin flexible instruments in which precise automatic manipulators are necessary [3]. These flexible manipulators exhibit important advantages in comparison to rigid ones, such as reduce energy consumption, smaller actuators, safety for humans, and more agility. Moreover, the effects of the collision with obstacles or humans are decreased because of the flexibility of the links. In spite of these advantages, the control problem of the flexible link manipulators becomes more complicated because the structural flexibility has to be controlled in such a way that the vibrations are cancelled if a good tip position and/or force control is to be gained.
Most of the work in the field of elastic robotic manipulator is confined to theoretical investigations. For validation the theoretical results by experimental testbed, it can be pointed out to the works of Cannon and Schmitz [4] as a pioneer in experimental study of single-link elastic robotic manipulator. Naganathan and Soni [5] also prepared an experimental setup composed of a single-link mounted on a stepper motor to verify their FEM results. Bellezza et al. [6] conducted experiments to study quasi-clamped and quasi-pinned end conditioned in slewing links. Luo [7] used a strain gauge sensor to measure the flexible robot arm deflections. Yang [8] presented a modal data-based method to estimate the dynamic response of an elastic robotic manipulator. Martins et al. [9] conducted experiments for a single-link elastic robotic manipulator to validate their suggested FEM based analytical model. More experimental testbed for vibration control of elastic robotic arm can be found in the investigation of Ladkany [10] and Peza Solis et al. [11].

So study and research on deflections and vibration of flexible link robots is an important problem. Increase of accuracy of measurements with finite number of sensors can help us. Flexible link's shapes can be approximated with different functions each one has specific nature. The usage of the best function that correspond nature of flexible link helps us to reduce sensor numbers and increases accuracy. 
Research on dynamics and control of flexible robots are dependent on monitoring of link situations. For this purpose many types of sensors can be used such as strain gauges, piezoceramics, accelerometer, and so forth.

\section{Kinematics of the Single-Link Elastic Robotic Manipulator}

The single-link flexible manipulator system considered in this work is shown in Figure 1, where $X Y Z$ and $x y z$ represent the stationary and moving coordinate's frames, respectively.

The position vector of arbitrary differential element $Q$ with respect to the local reference system $x y z$ is shown by $\vec{r}_{\mathrm{Q} / \mathrm{o}}$. The approach of modal analysis is used to incorporate the deflection of the link. So,

$$
\vec{r}_{\mathrm{Q} / o}=\vec{\eta}+\left\{\begin{array}{lll}
u & v & w
\end{array}\right\}^{T}
$$

where $\vec{\eta}=\left\{\begin{array}{lll}\eta & 0 & 0\end{array}\right\}^{T}$ is the position vector of differential element $Q$ with respect to $o$, when the elastic link is unreformed and $u, v$, and $w$ are small displacements in $o x, o y$, and $o z$ directions, respectively. To express these small displacements, the approach of assumed mode method is used as

$$
\left\{\begin{array}{lll}
u & v & w
\end{array}\right\}^{T}=\sum_{i=1}^{m} \delta_{i}(t) \vec{r}_{i}(\eta) .
$$

Here $\vec{r}_{i}=\left\{\begin{array}{lll}x_{i} & y_{i} & z_{i}\end{array}\right\}^{T}$ is the Eigen function vector whose components $x_{i}, y_{i}$, and $z_{i}$ are $i$ th longitudinal and transverse mode shapes of the link; $\delta_{i}$ is the $i$-th time-dependent modal generalized coordinate of the link, and $m$ is the number of modes used to define the deflection of the link.

The total transverse displacement of the centerline of differential element $Q$ is due to bending and shear. So the total slopes of the deflected centerline about $o y$ and $o z$ directions due to bending and shear deformation can be represented as

$$
\begin{gathered}
-\frac{\partial w}{\partial \eta}=\varphi_{y}+\theta_{y}, \\
\frac{\partial v}{\partial \eta}=\varphi_{z}+\theta_{z},
\end{gathered}
$$

where $\varphi_{y}$ and $\varphi_{z}$ are the slope of the deflected centerline due to shear and $\theta_{y}, \theta_{z}$ are the slope of the deflected centerline due to bending. Shear has no influence on rotating the differential element $Q$, and this differential element undertakes rotations only due to bending and torsion. So the rotation of this element in $o x, o y$, and $o z$ directions can be considered as $\theta_{x}$, $\theta_{y}$, and $\theta_{z}$, respectively. These small angles can be represented by truncated modal expansion as

$$
\vec{\theta}=\left\{\theta_{x} \theta_{y} \theta_{z}\right\}^{T}=\sum_{i=1}^{m} \delta_{i}(t) \vec{\theta}_{i}(\eta),
$$

where $\vec{\theta}_{i}=\left\{\begin{array}{lll}\theta_{x i} & \theta_{y i} & \theta_{z i}\end{array}\right\}^{T}$ is the Eigen function vector whose components $\theta_{x i}, \theta_{y i}$, and $\theta_{z i}$ are $i$-th rotational mode shapes of the link in $o x, o y$, and $o z$ directions, respectively.

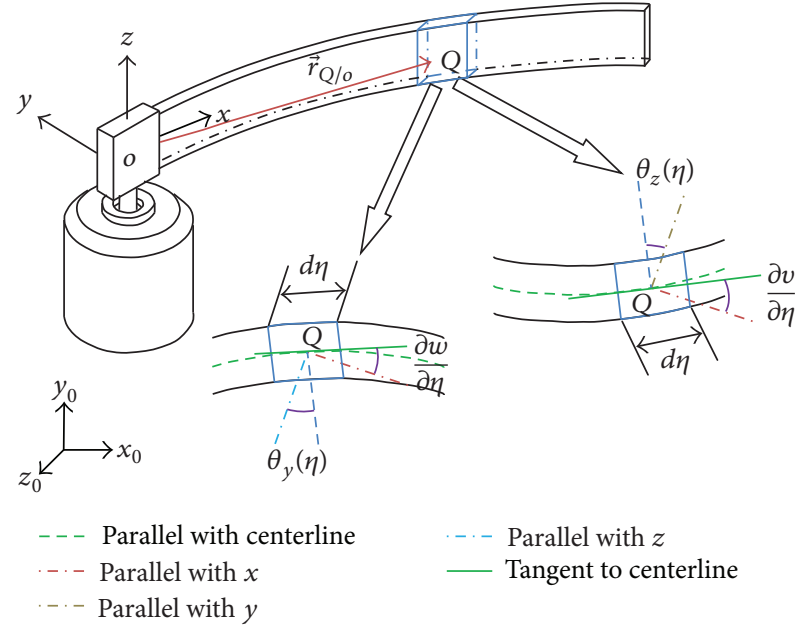

FIGURE 1: Single-link elastic robotic manipulator.

\section{The System Gibbs Function and Its Derivatives}

In this section the system's acceleration energy and its derivatives with respect to quasi-accelerations are developed for construction the G-A formulation. With the assumption of TBT, the acceleration energy of a differential element $Q$ can be represented as

$$
d s=\frac{1}{2} \mu(\eta)\left(\ddot{\vec{r}}_{Q}^{T} \cdot \ddot{\vec{r}}_{Q}\right) d \eta+\frac{1}{2} \ddot{\vec{\theta}}^{T} \cdot J(\eta) \ddot{\vec{\theta}} d \eta .
$$

But with the assumption of EBBT, only the first term of (5) should be preserved. Also $\mu(\eta)$ and $J(\eta)$ are mass per unit length and mass moment of inertia per unit length, respectively. Note that $\ddot{\vec{r}}_{Q}$ and $\ddot{\vec{\theta}}$ are linear and angular acceleration of differential element $Q$. These two terms can be stated as

$$
\begin{gathered}
\ddot{\vec{r}}_{\mathrm{Q}}=\ddot{\vec{r}}_{\mathrm{Q} / o}+2 \vec{\omega} \times \dot{\vec{r}}_{\mathrm{Q} / o}+\dot{\vec{\omega}} \times \vec{r}_{\mathrm{Q} / o}+\vec{\omega} \times\left(\vec{\omega} \times \vec{r}_{\mathrm{Q} / o}\right), \\
\ddot{\vec{\theta}}=\sum_{j=1}^{m} \ddot{\delta}_{j}(t) \vec{\theta}_{j}(\eta) .
\end{gathered}
$$

In the above expressions, $\vec{\omega}$ and $\dot{\vec{\omega}}$ are angular velocity and angular acceleration of the link, respectively. Also $\dot{\vec{r}}_{\mathrm{Q} / o}$ and $\ddot{\vec{r}}_{\mathrm{Q} / \mathrm{o}}$ are velocity and acceleration of differential element $Q$ with respect to the origin of the local reference system, respectively. Inserting (6) into (5) and integrating over the link from 0 to $l$, the total acceleration energy of the link will be obtained as

$$
\begin{aligned}
S= & \frac{1}{2} B_{1}-2 \vec{\omega}^{T} \cdot \vec{B}_{2}+\dot{\vec{\omega}}^{T} \cdot \vec{B}_{3}-\vec{\omega}^{T} \cdot B_{4} \vec{\omega}+2 \dot{\vec{\omega}}^{T} \cdot B_{5} \vec{\omega} \\
& +\frac{1}{2} \dot{\vec{\omega}}^{T} \cdot B_{6} \dot{\vec{\omega}}+\dot{\vec{\omega}}^{T} \cdot \widetilde{\omega} B_{6} \vec{\omega}+\frac{1}{2} B_{7}+\text { irrelevant terms }
\end{aligned}
$$

where $\widetilde{\omega}$ is the skew-symmetric tensor associated with $\vec{\omega}$ vector. Also there is a term named as "irrelevant terms." 
Motion equation with G-A formulation will be constructed by taking the partial derivatives of Gibbs' function with respect to quasi-accelerations. So, the term in Gibbs' function that does not contain $\ddot{q}$ and $\ddot{\delta}_{j}$ can be ignored. The variables that appeared in (7) can be calculated as,

$$
\begin{aligned}
& B_{1}=\sum_{j=1}^{m} \sum_{k=1}^{m} \ddot{\delta}_{j} \ddot{\delta}_{k} C_{1 j k}, \\
& \vec{B}_{2}=\sum_{j=1}^{m} \sum_{k=1}^{m} \ddot{\delta}_{j} \dot{\delta}_{k} \vec{C}_{2 j k}, \\
& \vec{B}_{3}=\sum_{j=1}^{m} \ddot{\delta}_{j} \vec{\alpha}_{j}, \\
& B_{4}=\sum_{j=1}^{m} \ddot{\delta}_{j} \beta_{j}, \\
& B_{5}=\sum_{j=1}^{m} \dot{\delta}_{j} \beta_{j}, \\
& B_{6}=C_{3}+\sum_{j=1}^{m} \delta_{j}\left(C_{4 j}^{T}+\beta_{j}\right), \\
& B_{7}=\sum_{j=1}^{m} \sum_{k=1}^{m} \ddot{\delta}_{j} \ddot{\delta}_{k} C_{5 j k},
\end{aligned}
$$

where

$$
\begin{aligned}
C_{1 j k} & =\int_{0}^{l} \mu \vec{r}_{j}^{T} \cdot \vec{r}_{k} d \eta, \\
\vec{C}_{2 j k} & =\int_{0}^{l} \mu \widetilde{r}_{j} \vec{r}_{k} d \eta, \\
C_{3} & =\int_{0}^{l} \mu \tilde{\eta}^{T} \widetilde{\eta} d \eta, \\
C_{4 j} & =\int_{0}^{l} \mu \widetilde{\eta}^{T} \widetilde{r}_{j} d \eta, \\
C_{5 j k} & =\int_{0}^{l} \vec{\theta}_{j}^{T} \cdot J \vec{\theta}_{k} d \eta, \\
\vec{C}_{6 j} & =\int_{0}^{l} \mu \widetilde{\eta}^{r_{j}} d \eta, \\
C_{j k} & =\int_{0}^{l} \mu \vec{r}_{j}^{T} \widetilde{r}_{k} d \eta, \\
\beta_{j} & =C_{4 j}+\sum_{k=1}^{m} \delta_{k} C_{7 k j} . \\
\vec{\alpha}_{j} & =\sum_{k=1}^{m} \delta_{k} \vec{C}_{2 k j},
\end{aligned}
$$

In the above equations, $\widetilde{\eta}$ and $\widetilde{r}_{j}$ are skew-symmetric tensors associated with $\vec{\eta}$ and $\vec{r}_{j}$ vectors. As mentioned above, one part of dynamic equations of the system using (G-A) formulation will be obtained by differentiating of Gibbs' function with respect to quasi-accelerations. These two terms can be represented as

$$
\begin{gathered}
\frac{\partial S}{\partial \ddot{q}}=\frac{\partial \dot{\vec{\omega}}^{T}}{\partial \ddot{q}} \cdot\left(\vec{B}_{3}+2 B_{5} \vec{\omega}+B_{6} \dot{\vec{\omega}}+\widetilde{\omega} B_{6} \vec{\omega}\right), \\
\frac{\partial S}{\partial \ddot{\delta}_{j}}=\sum_{k=1}^{m} \ddot{\delta}_{k}\left(C_{1 j k}+C_{5 j k}\right)-2 \vec{\omega}^{T} \cdot \sum_{k=1}^{m} \dot{\delta}_{k} \vec{C}_{2 j k}-\vec{\omega}^{T} \cdot \beta_{j} \vec{\omega} \\
+\dot{\vec{\omega}}^{T} \cdot \vec{\alpha}_{j} .
\end{gathered}
$$

\section{The System's Potential Energy and Its Derivatives}

The potential energy of the system arises from two sources, first potential energy due to gravity and second potential energy due to the elastic deformations. The corresponding energy due to the gravity can be considered simply by inserting $\ddot{\vec{r}}_{O}=\vec{g}$, where $\vec{g}$ is the acceleration of gravity.

To express the strain potential energy stored in elastic link, let us assume two theories hold that: TBT and EBBT. For the first assumption the strain potential energy will be expressed in terms of deflections and rotations as

$$
\begin{aligned}
V_{e}=\frac{1}{2} \int_{0}^{l} & {\left[k \mathrm{AG}\left(\varphi_{y}{ }^{2}+\varphi_{z}{ }^{2}\right)+E I_{y}\left(\frac{\partial \theta_{y}}{\partial \eta}\right)^{2}+E I_{z}\left(\frac{\partial \theta_{z}}{\partial \eta}\right)^{2}\right.} \\
& \left.+E \mathrm{~A}\left(\frac{\partial u}{\partial \eta}\right)^{2}+G I_{x}\left(\frac{\partial \theta_{x}}{\partial \eta}\right)^{2}\right] d \eta
\end{aligned}
$$

But for the second assumption, the first term in the above integral will be omitted. In (11), $E$ and $G$ are modulus of elasticity and shear modulus, respectively; $I_{x}$ is the polar area moment of inertia about $o x$ axis; $I_{y}$ and $I_{z}$ are area moment of inertia about $o y$ and $o z$ axes, respectively; $A$ is the cross section area of the link and $k$ is shear correction factor.

As noted in the previous section the small angles $\theta_{x}$, $\theta_{y}, \theta_{z}$ and small displacements $u, v, w$ can be expressed with a truncated modal approximation. By inserting these expressions in (11), the strain potential energy for the link will be obtained as

$$
V_{e}=\frac{1}{2} \sum_{j=1}^{m} \sum_{k=1}^{m} \delta_{j}(t) \delta_{k}(t) K_{j k} \text {, }
$$


where

$$
\begin{aligned}
K_{j k}=\int_{0}^{l}[ & k \mathrm{~A} G\left(\varphi_{y j} \varphi_{y k}+\varphi_{z j} \varphi_{z k}\right)+E I_{y} \frac{\partial \theta_{y j}}{\partial \eta} \frac{\partial \theta_{y k}}{\partial \eta} \\
& +E I_{z} \frac{\partial \theta_{z j}}{\partial \eta} \frac{\partial \theta_{z k}}{\partial \eta}+E \mathrm{~A} \frac{\partial x_{j}}{\partial \eta} \frac{\partial x_{k}}{\partial \eta} \\
& \left.+G I_{x} \frac{\partial \theta_{x j}}{\partial \eta} \frac{\partial \theta_{x k}}{\partial \eta}\right] d \eta .
\end{aligned}
$$

For deriving the equations of motion of viscoelastic robotic manipulators, the partial derivatives of strain potential energy with respect to generalized coordinates are needed. These two terms can be represented as

$$
\begin{gathered}
\frac{\partial V_{e}}{\partial q}=0, \\
\frac{\partial V_{e}}{\partial \delta_{j}}=\sum_{k=1}^{m} \delta_{k}(t) K_{k j} .
\end{gathered}
$$

\section{Rayleigh's Dissipation Function and Its Derivatives}

An important class of nonconservative forces is the class of forces that dissipate energy. A common way of considering energy-dissipating forces is the assumption of viscous damping. In this model, the viscous damping force is modeled as a force opposing the velocity and proportional to it. The special case of linear proportionality is commonly used, because it is a linear approximation and easier to deal with, mathematically. This approximation is used especially when modeling light amounts of damping. In analytical mechanics, a convenient way of treating viscous damping forces is the use of Rayleigh's dissipation function. Rayleigh's dissipation function associated with these internal and external damping for the flexible link can be represented as

$$
\begin{aligned}
D= & \frac{1}{2} \int_{0}^{l} \gamma\left[\left(\frac{\partial v}{\partial t}\right)^{2}+\left(\frac{\partial w}{\partial t}\right)^{2}\right] d \eta \\
& +\frac{1}{2} \int_{0}^{l_{i}} K_{v}\left[I_{z}\left(\frac{\partial^{3} v}{\partial \eta^{2} \partial t}\right)^{2}+I_{y}\left(\frac{\partial^{3} w}{\partial \eta^{2} \partial t}\right)^{2}\right] d \eta,
\end{aligned}
$$

where $K_{v}$ is the Kelvin-Voigt damping coefficient of the elastic link, and $\gamma$ is the air damping coefficient. Inserting (2) into (15), Rayleigh's dissipation function for the system will be obtained as

$$
D=\frac{1}{2} \sum_{j=1}^{m} \sum_{k=1}^{m} \dot{\delta}_{j}(t) \dot{\delta}_{k}(t) D_{j k}
$$

where

$$
\begin{aligned}
D_{j k}= & \int_{0}^{l} \gamma\left(y_{j} y_{k}+z_{j} z_{k}\right) d \eta \\
& +\int_{0}^{l} K_{v}\left(I_{z} \frac{\partial^{2} y_{j}}{\partial \eta^{2}} \frac{\partial^{2} y_{k}}{\partial \eta^{2}}+I_{y} \frac{\partial^{2} z_{j}}{\partial \eta^{2}} \frac{\partial^{2} z_{k}}{\partial \eta^{2}}\right) d \eta .
\end{aligned}
$$

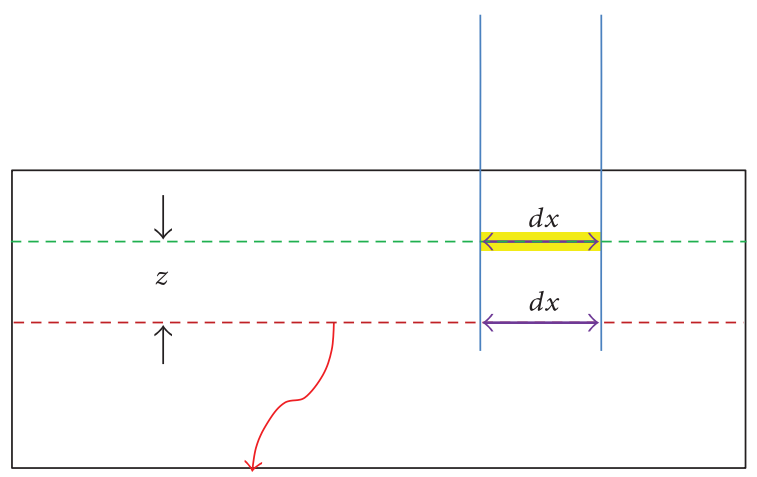

Neutral axis

Figure 2: Differential element before bending.

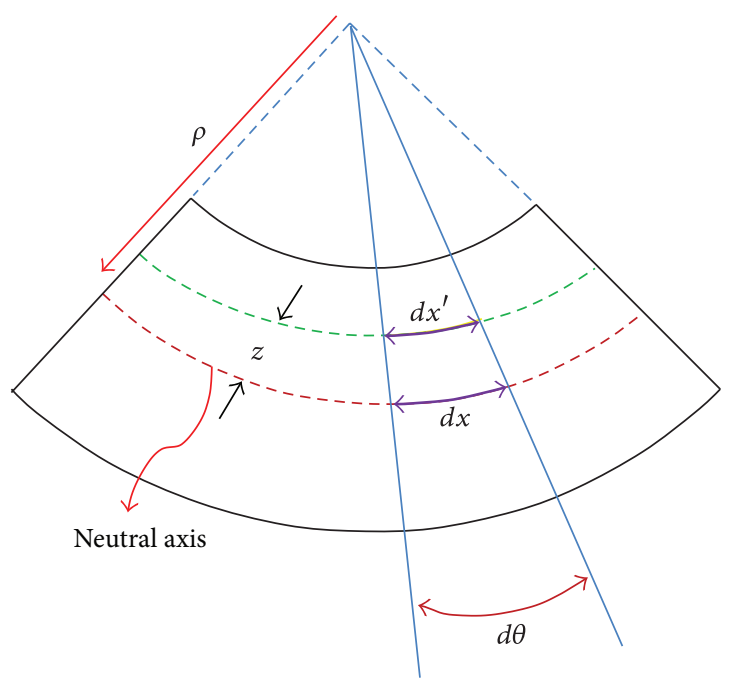

Figure 3: Differential element after bending.

Generalized forces due to internal and external damping are obtained by taking partial derivatives of Rayleigh's dissipation function with respect to generalized velocities. These two terms may be presented as

$$
\begin{gathered}
\frac{\partial D}{\partial \dot{q}}=0, \\
\frac{\partial D}{\partial \dot{\delta}_{j}}=\sum_{k=1}^{m} \dot{\delta}_{k}(t) D_{k j} .
\end{gathered}
$$

\section{Dynamic Equations of Flexible Link Manipulator}

Motion equation of viscoelastic robotic manipulators will be completed by considering the generalized forces which are caused by the remaining external force terms. Let us assume that there is no external load on the links of the considered robotic manipulator. So, the generalized forces in the deflection equations will be zero. The generalized force in the joint equations is the torque $\tau$ that applies to the joint. 


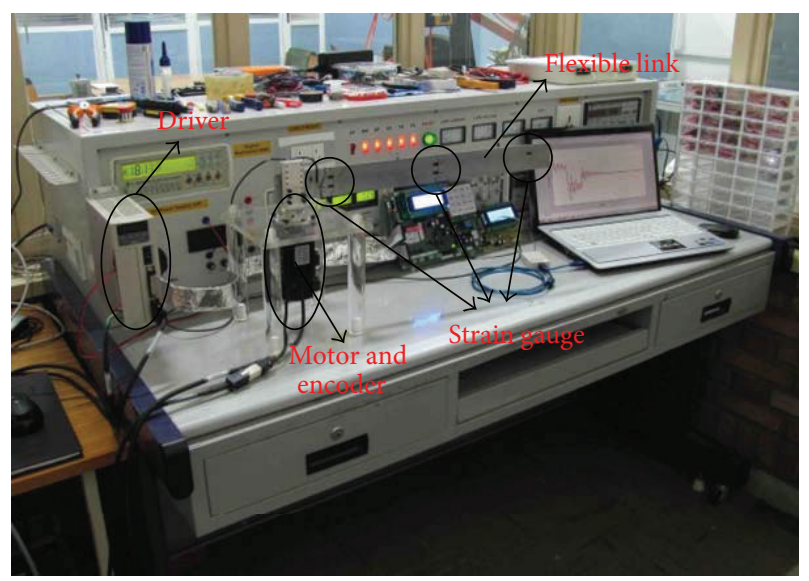

FIgURE 4: The IUST flexible manipulator.

With this assumption, the dynamic equations of motion by (G-A) formulation will be completed as follows

(i) The joint equations of motion:

$$
\frac{\partial S}{\partial \ddot{q}}+\frac{\partial D}{\partial \dot{q}}+\frac{\partial V_{e}}{\partial q}=\tau .
$$

(ii) The deflection equations of motion:

$$
\frac{\partial S}{\partial \ddot{\delta}_{j}}+\frac{\partial D}{\partial \dot{\delta}_{j}}+\frac{\partial V_{e}}{\partial \delta_{j}}=0 \quad j=1,2, \ldots, m .
$$

6.1. Strain and Curvature. If the strain in different sections of an elastic beam is known, the shape of the beam can be estimated by interpolation. The differential element in yellow color in Figures 2 and 3 shows the beam's neutral axis before and after bending, respectively.

The strain of this differential element can be presented as:

$$
\varepsilon(x)=\frac{(\rho-z) \cdot d \theta-\rho \cdot d \theta}{\rho \cdot d \theta}=-\frac{z}{\rho} .
$$

Based on (21), the strain on the surface of the beam will be obtained as

$$
\varepsilon(x)=-\frac{t}{2 \rho(x)},
$$

where $t$ is the thickness of the beam. On the other hand from elementary calculus the curvature of a plane curve can be expressed as:

$$
\frac{1}{\rho}=\frac{y^{\prime \prime}(x)}{\left(1+y^{\prime}(x)^{2}\right)^{3 / 2}} .
$$

Thus, the strain of the beam in terms of the function $y(x)$ can be represented as:

$$
\varepsilon(x)=-\frac{t \cdot y^{\prime \prime}(x)}{2\left(1+y^{\prime}(x)^{2}\right)^{3 / 2}} .
$$

But, in the case of the elastic curve of a beam, the slope $y^{\prime}$ is very small, and its square is negligible compared to unity. So,

$$
\varepsilon(x)=-\frac{t \cdot y^{\prime \prime}(x)^{2}}{2} .
$$

Therefor, the strain is a function of curvature and thickness. The magnitude of strain in $x$ position will be obtained with a strain gauge on that point. As we cannot use infinite number of sensors, the strain is definite only in finite points. We can guess $y(x)$ as a suitable function that can approximate link's shape. The nature of the function must be the same with the nature of the flexible link. If one uses suitable function for link's shape, fewer constants and fewer sensors will be needed. In this paper, the following functions will be used:

$$
\begin{aligned}
& A \cdot x^{4}+B \cdot x^{3}+C \cdot x^{2}+E \cdot x+F, \\
& A \cdot x^{6}+B \cdot x^{5}+C \cdot x^{4}+D \cdot x^{3}+E \cdot x^{2}+F \cdot x+G, \\
& A \cdot \sin (x)+B \sin (2 \cdot x)+C \cdot \cos (x)+D \cdot \cos (2 \cdot x)+E, \\
& A \cdot \sin (x)+B \cdot \sin (2 \cdot x)+C \cdot \sin (3 \cdot x)+D \cdot \cos (x) \\
& +E \cdot \cos (2 \cdot x)+F \cdot \cos (3 \cdot x)+G, \\
& A \cdot \sinh (x)+B \cdot \sinh (2 \cdot x)+C \cdot \cosh (x) \\
& +D \cdot \cosh (2 \cdot x)+E \text {, } \\
& A \cdot \sinh (x)+B \cdot \sinh (2 \cdot x)+C \cdot \sinh (3 \cdot x) \\
& +D \cdot \cosh (x)+E \cdot \cosh (2 \cdot x) \\
& +F \cdot \cosh (3 \cdot x)+G \text {. }
\end{aligned}
$$

In experimental setup, three full bridge strain gauges attached in three different positions. By the use of (25) and considering the three strain gauge bridges, we can organize three equations. In addition, from boundary condition, slope and deflection at the beginning of the link are zero. So, $y$ and $y^{\prime}$ in this point will be zero. With these three strains and two boundary conditions, five equations with five unknowns can be solved. But if we consider the boundary conditions at the end point of the link, two more equations will be added. In fact at the end point of the link shear and moment are zero. So, $y^{\prime \prime}$ and $y^{\prime \prime \prime}$ will be zero in this point. In this condition, we have seven equations with seven unknowns and we can guess a function with more constants and more accuracy.

\section{Experimental Setup}

The experimental testbed is the flexible manipulator that is prepared at the Department of Mechanical Engineering of the Iran University of Science and Technology Figure 4. The experimental setup consists of a single-link flexible manipulator driven by a servo-tech AC servo motor. This $400 \mathrm{~W}$ AC servomotor can be drived in position, speed, and toque mode. In order to remove additional effect of bearing friction, the flexible manipulator is directly attached to 


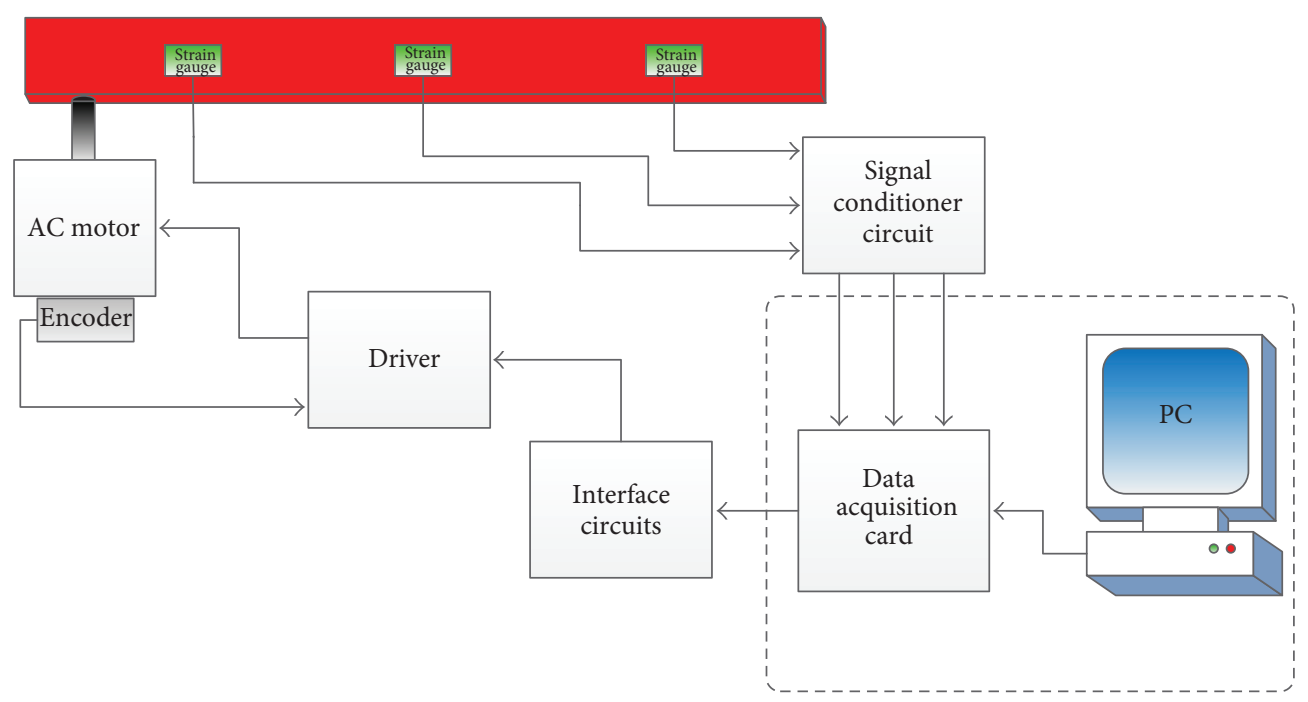

FIGURE 5: Schematic view of the experimental setup.

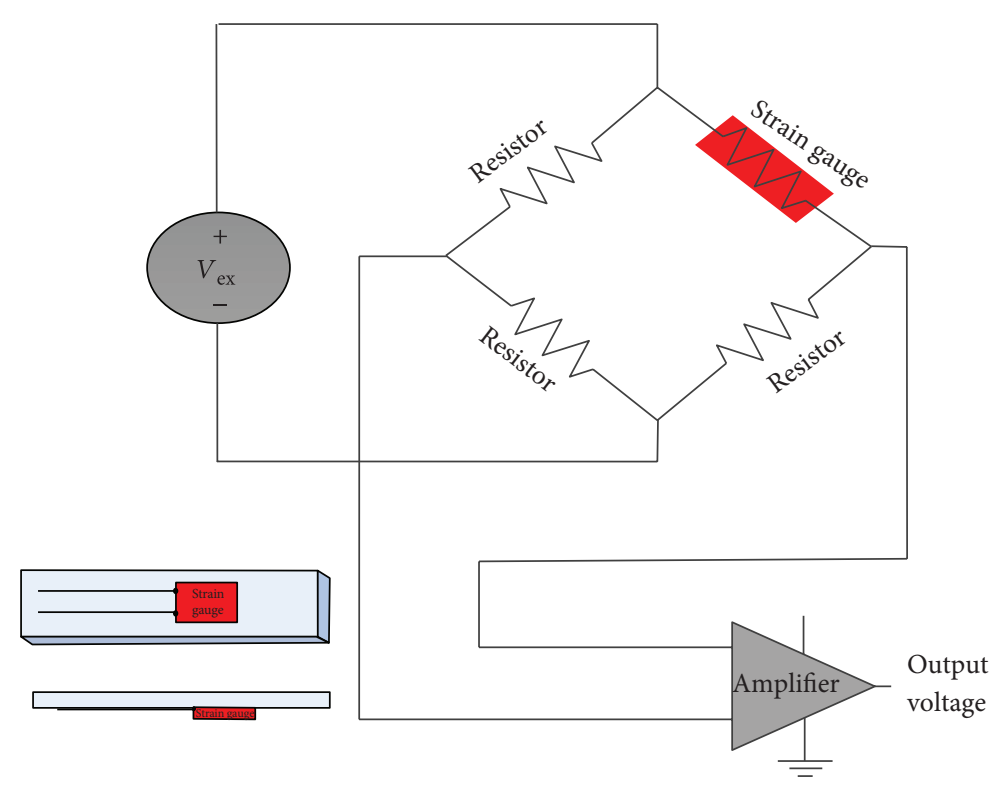

FIGURE 6: Quarter bridge layout for strain gauges.

the motor shaft. In Table 1 the physical properties of the flexible manipulator are summarized. Also, the Schematic diagram of the experimental setup is shown in Figure 5.

The flexible manipulator has a planar motion thus the effect of gravity can be ignored. An incremental encoder with the resolution of 2500 pulse per revolution is used to calculate the rotation angle of the motor. Motor angular velocity is determined by time derivation of the motor angular position data. The tip deflection and vibration of the link are measured by three strain gauge bridges mounted on the link. The analogue signals of the strain gauges amplify through a signal conditioner circuit and sent to a data acquisition card.
A 64-bit personal computer was used for control motor output via an Advantech PCI-1710HG data acquisition card. PCI-1710HG is a multifunction card with 12 bits resolution and $100 \mathrm{kS} / \mathrm{s}$ sampling rate.

Strain gauges can be attached in many ways for different purposes. For recording vibrations without temperature compensation, we have three-type layouts. Quarter bridge, half bridge and full bridge layout can be used for this purpose. Quarter bridge (Figure 6) has less sensitivity than two other types. So, it can be economic. But the weakness of signals makes the necessity of expensive signal conditioners. 


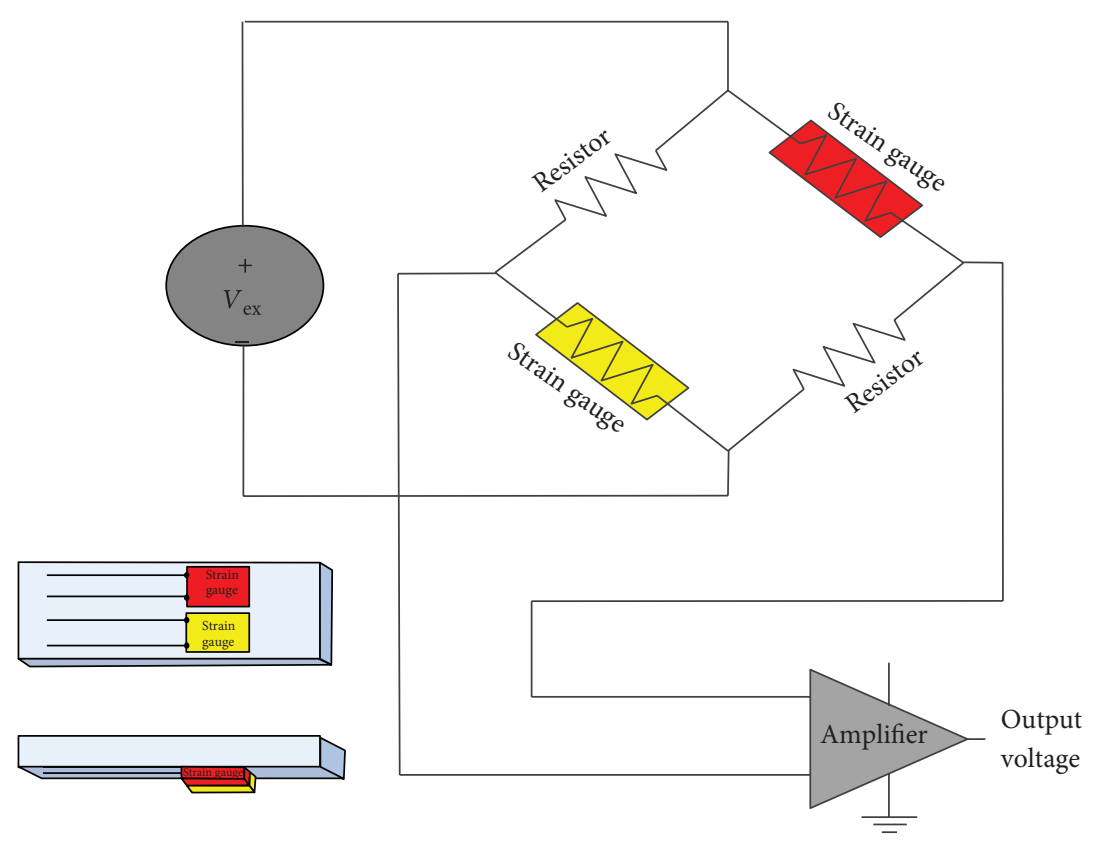

Figure 7: Half bridge layout for strain gauges.

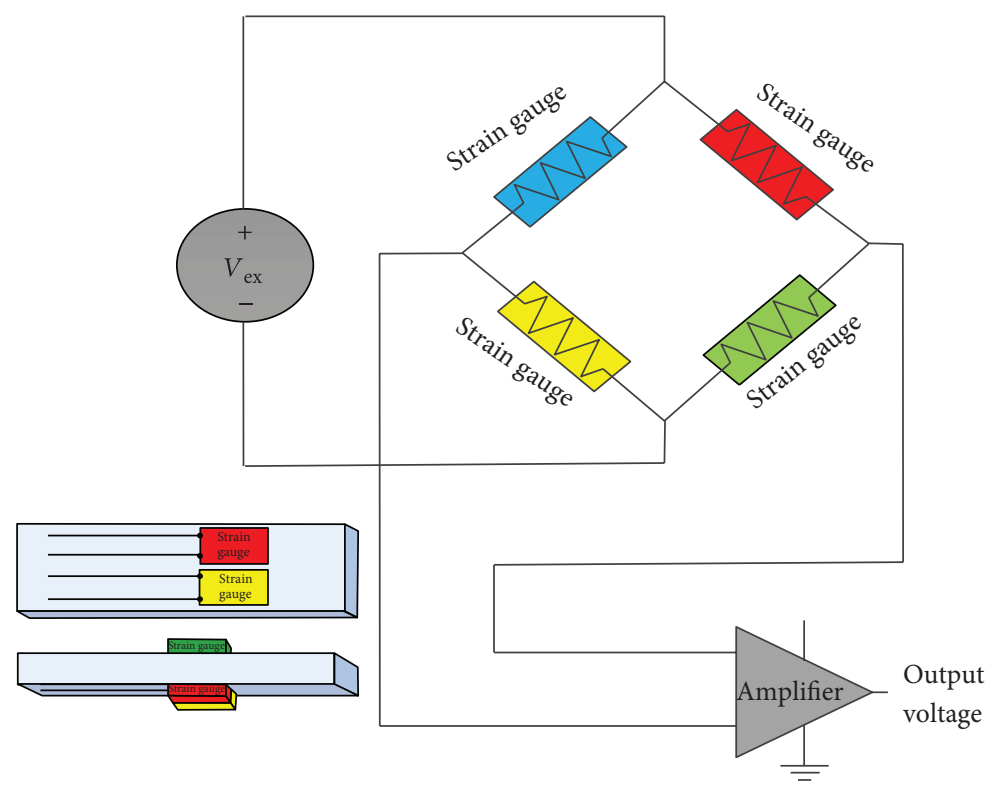

FIGURE 8: Full bridge layout for strain gauges.

Half bridge (Figure 7) and full bridge layouts have more sensitivity but need more number of sensors, respectively. Half bridge layout uses two strain gauges, and if it is required, two other strain gauges in normal directions can be added in order to compensate the temperature effects.

Full bridge layout (Figure 8) uses four strain gauges and has best sensitivity and simplest equation. But as the bridge is full, it is not possible to use temperature compensator in this type.

The following equations can be used for calculation the exact magnitude of strain:

$$
\begin{aligned}
\frac{V_{o}}{V_{\mathrm{Ex}}}=-\frac{G F \cdot \varepsilon}{4}\left(\frac{1}{1+G F \cdot(\varepsilon / 2)}\right), \\
\frac{V_{o}}{V_{\mathrm{Ex}}}=-\frac{G F \cdot \varepsilon}{2}, \\
\frac{V_{o}}{V_{\mathrm{Ex}}}=-G F \cdot \varepsilon .
\end{aligned}
$$

These three equations show relations between $V_{o}$ and strain in quarter bridge, half bridge, and full bridge, respectively. It is clear that quarter bridge equation has nonlinear 


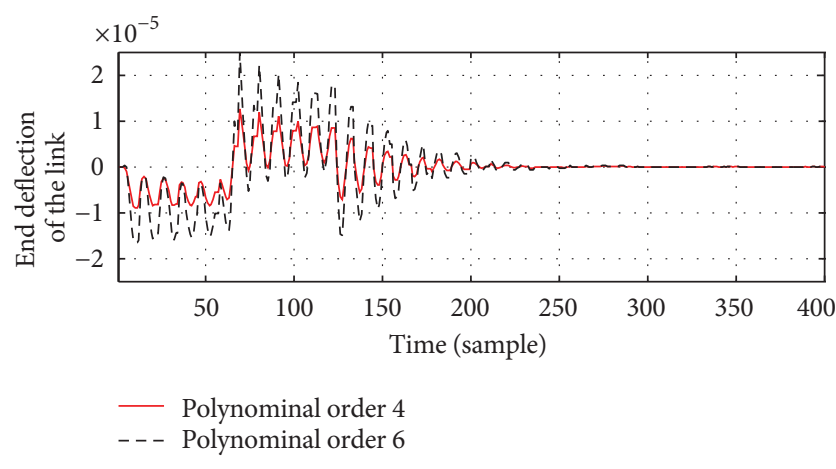

FIGURE 9: Estimation of end point deflection with 6th-order and 4th-order polynomials.

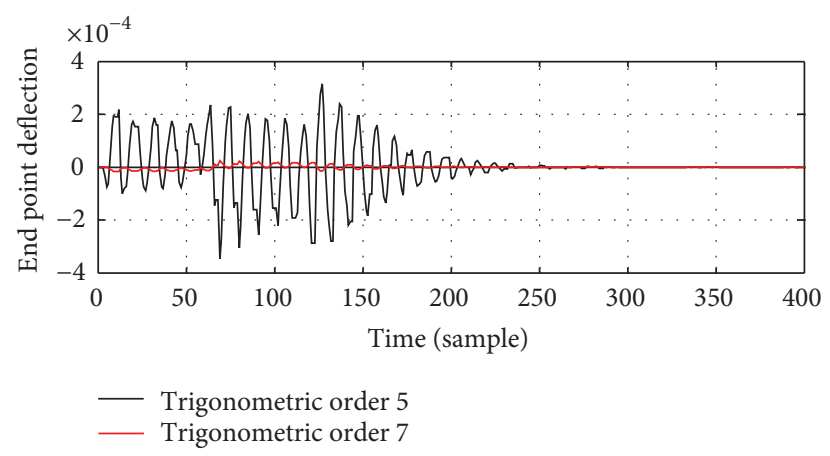

FIGURE 10: Estimation of end point deflection with 5 th and 7 th order trigonometric functions.

form, but the other two types have linear and simple relations. In the above equations $V_{\mathrm{Ex}}$ is excitation voltage exerted on the Wheatstone bridge. $V_{o}$ in these equations exhibit output voltage. Excitation voltage affects $V_{o}$. On the other hand, increasing in excitation voltage causes increasing in output voltage and provides more resolution. But increasing in exoitation voltage causes increasing in ampere. It can generates heat in strain gauges. Generated heat in strain gauges must be compensated with temperature compensator strain gauges. Otherwise, error increases in output voltage.

\section{Results and Discussion}

In this section, simulation and experimental results of the response of a flexible manipulator and the effect of functions are presented. The torque input implemented to the manipulator is a bang-bang torque with amplitude of $\pm 0.13 \mathrm{~N} \cdot \mathrm{m}$ and duty cycle of $0.3 \mathrm{~s}$. This torque has a positive (acceleration) and a negative (deceleration) period allowing the manipulator to initially accelerates and then decelerates and eventually stops at a target location. There are 400 data from each strain gauge bridges that can help to have a comparison between experimental results and theoretical results. Sampling frequency is $200 \mathrm{~Hz}$.

At first, we compare a sixth-order polynomial with a fourth-order polynomial.
TABLE 1: Parameters of the flexible link.

\begin{tabular}{lcc}
\hline Number & Description & Value \\
\hline 1 & Length $(\mathrm{m})$ & 0.5 \\
2 & Height $(\mathrm{m})$ & 0.517 \\
3 & Thickness $(\mathrm{m})$ & 0.0015 \\
4 & Bending stiffness $\left(\mathrm{N} \cdot \mathrm{m}^{2}\right)$ & 1.01784 \\
5 & Hub inertia $\left(\mathrm{Kg} \cdot \mathrm{m}^{2}\right)$ & 0.0015 \\
6 & Shear modulus $\left(\mathrm{N} / \mathrm{m}^{2}\right)$ & $27 e 9$ \\
\hline
\end{tabular}

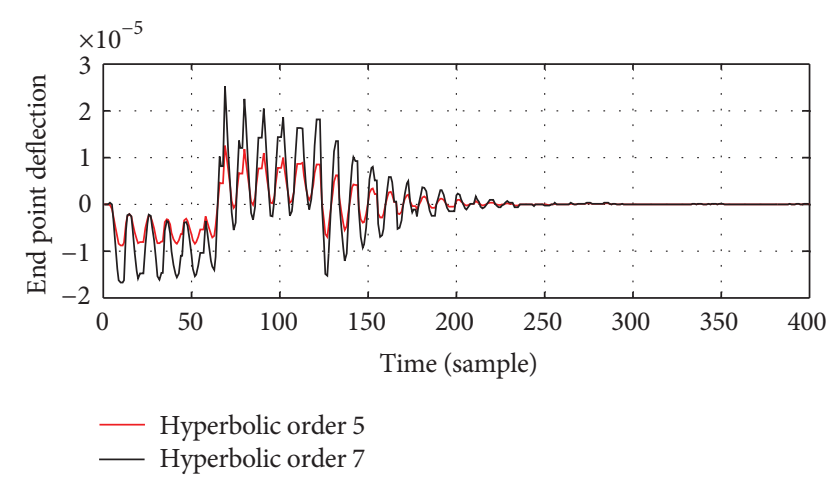

Figure 11: Comparison between 5th-and 7th, order hyperbolic functions.

It's clear in Figure 9, 6th-order polynomial exhibits better results from sensors data. If we have free conditions at the end of the link, it's better to use this function.

Figure 10 shows that the interpolation with 5 th-order trigonometric function leading to very bad results. The function has no enough agility for tracking vibrations. So the behavior of trigonometric functions is far from link vibrations.

At the next stage, the behavior of hyperbolic functions will be studied. As shown in Figure 11, comparison between hyperbolic functions with different constants shows similar results. Nevertheless, the use of hyperbolic function with seven constants yields better results.

In the next step comparison between trigonometric, hyperbolic, and polynomial functions with seven constants will be shown. As seen in Figure 12 vibration behavior of these functions is the same. Although the nature of these three functions is not similar, very good fitting will be observed.

Continuously experimental results will be compared with the Gibbs-Apple theoretical results. For experimental estimation, hyperbolic function with seven contents is used.

In Figure 13 experimental results follow theoretical results closely. So hyperbolic and polynomials are suitable for estimating flexible links vibrations.

\section{Conclusions}

In this paper the equation of motion of a single-like flexible manipulator is derived by the Gibbs-Appell formulation. Also an experimental test bed was prepared to verify the simulation results. The obtained results from strain gauges 


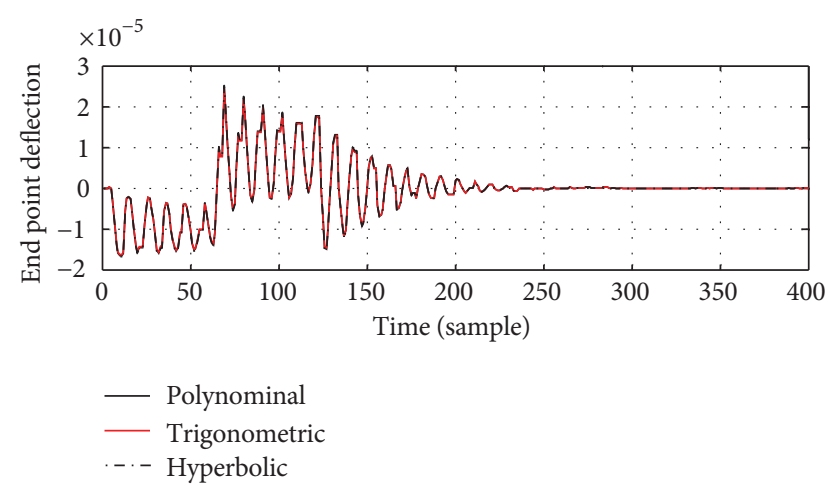

FiguRE 12: Comparison between polynomial, trigonometric, and hyperbolic functions with seven constants.

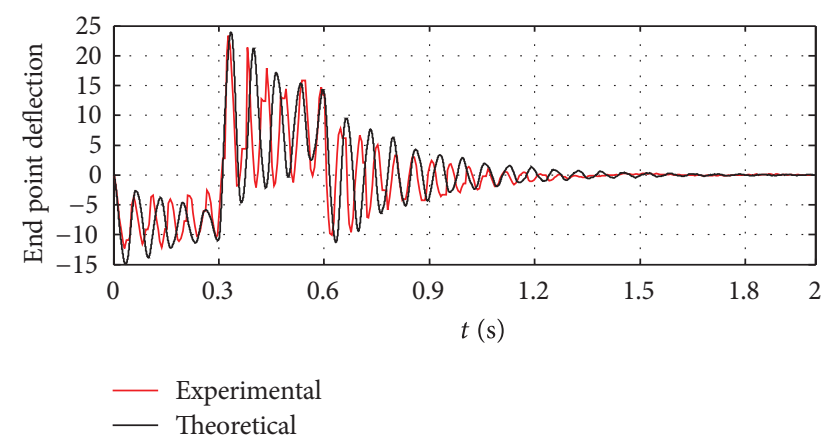

Figure 13: Experiment and theoretical result comparison.

converted to end point deflection by different functions. These functions can be used for monitoring flexible link vibrations. Among these functions, the obtained result from hyperbolic function follows theoretical results much better than the other functions.

\section{References}

[1] I. Payo, V. Feliu, and O. D. Cortázar, "Force control of a very lightweight single-link flexible arm based on coupling torque feedback," Mechatronics, vol. 19, no. 3, pp. 334-347, 2009.

[2] O. Sawodny, H. Aschemann, and A. Bulach, "Mechatronical designed control of fire-rescue turntable-ladders as flexible link robots," in Proceedings of the 15th Triennial World Congress International Federation of Automatic Control (IFAC '02), Barcelona, Spain, 2002.

[3] R. A. Beasley and R. D. Howe, "Model-based error correction for flexible robotic surgical instruments," in Proceedings of Robotics: Science and System Conference, vol. 1, 2005.

[4] R. H. Cannon and E. Schmitz, "Initial experiments on endpoint control of a flexible one-link robot," International Journal of Robotics Research, vol. 3, no. 3, pp. 62-75, 1984.

[5] G. Naganathan and A. H. Soni, "An analytical and experimental investigation of flexible manipulator performance," in Proceeding of the IEEE International Conference on Robotic and Automation, vol. 4, pp. 767-773, 1987.

[6] F. Bellezza, L. Lanari, and G. Ulivi, "Exact modeling of the flexible slewing link," in Proceedings of the IEEE International
Conference on Robotics and Automation, pp. 734-739, May 1990.

[7] Z. H. Luo, "Direct strain feedback control of flexible robot arms: new theoretical and experimental results," IEEE Transactions on Automatic Control, vol. 38, no. 11, pp. 1610-1622, 1993.

[8] Z. Yang, "Prediction of the dynamic response of flexible manipulators from a modal database," Mechanism and Machine Theory, vol. 32, no. 6, pp. 679-689, 1997.

[9] J. M. Martins, Z. Mohamed, M. O. Tokhi, J. Sá da Costa, and M. A. Botto, "Approaches for dynamic modelling of flexible manipulator systems," IEE Proceedings on Control Theory and Applications, vol. 150, no. 4, pp. 401-411, 2003.

[10] S. G. Ladkany, "The dynamic response of a flexible threelink robot using strain gages and lagrange polynomials," in Proceedings of the 4th World Conference on Robotics Research, 1991.

[11] J. F. Peza Solis, G. Silva Navarro, and R. Castro Linares, "Modeling tip position control of a flexible link robot," Computation y Sistemas, vol. 12, no. 4, pp. 421-435, 2008. 

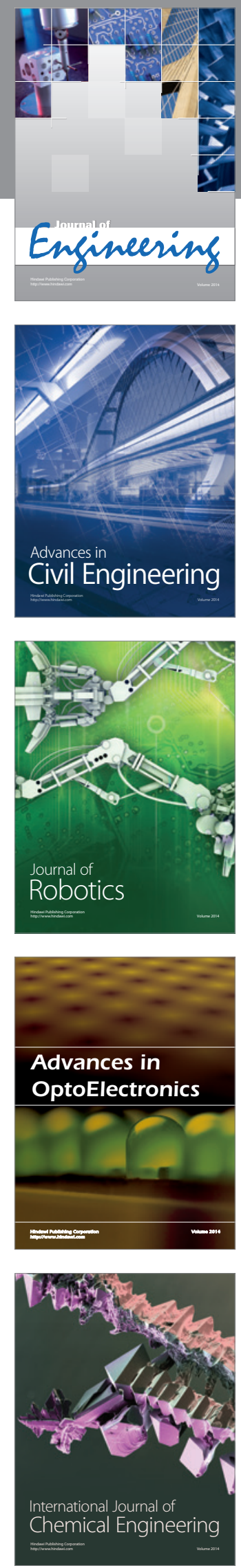

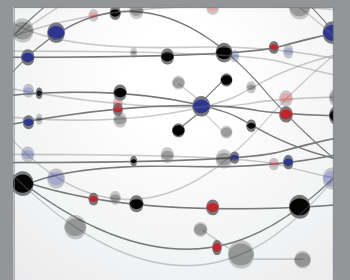

The Scientific World Journal
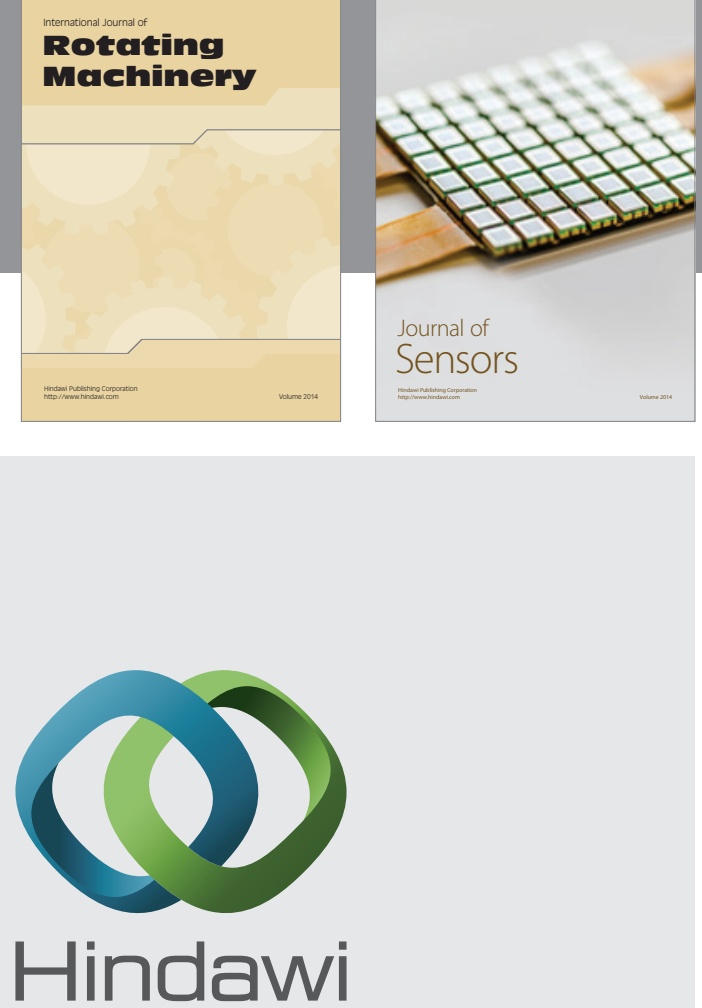

Submit your manuscripts at http://www.hindawi.com
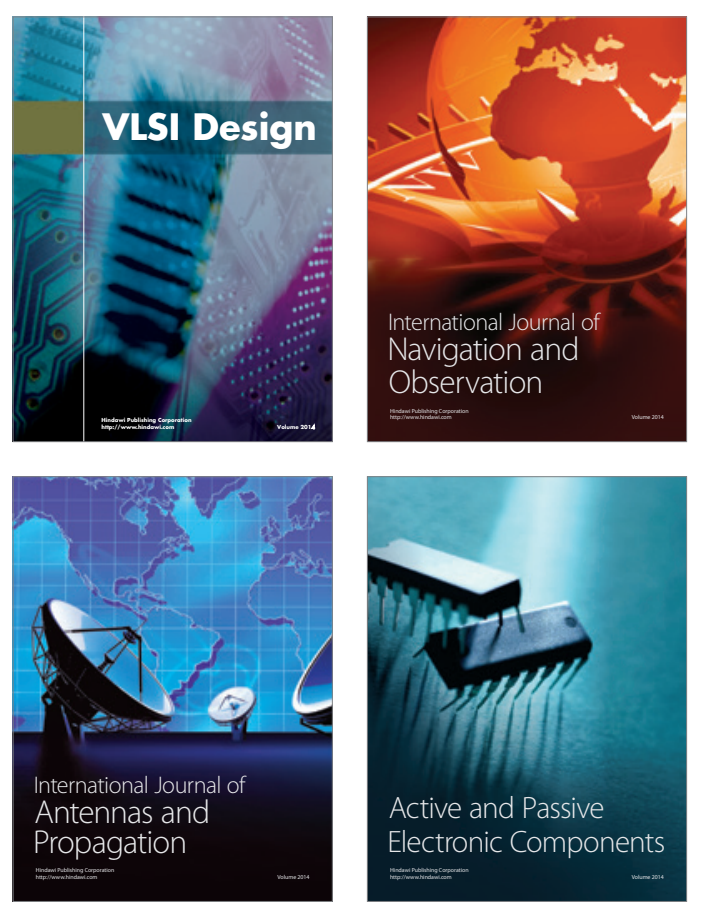
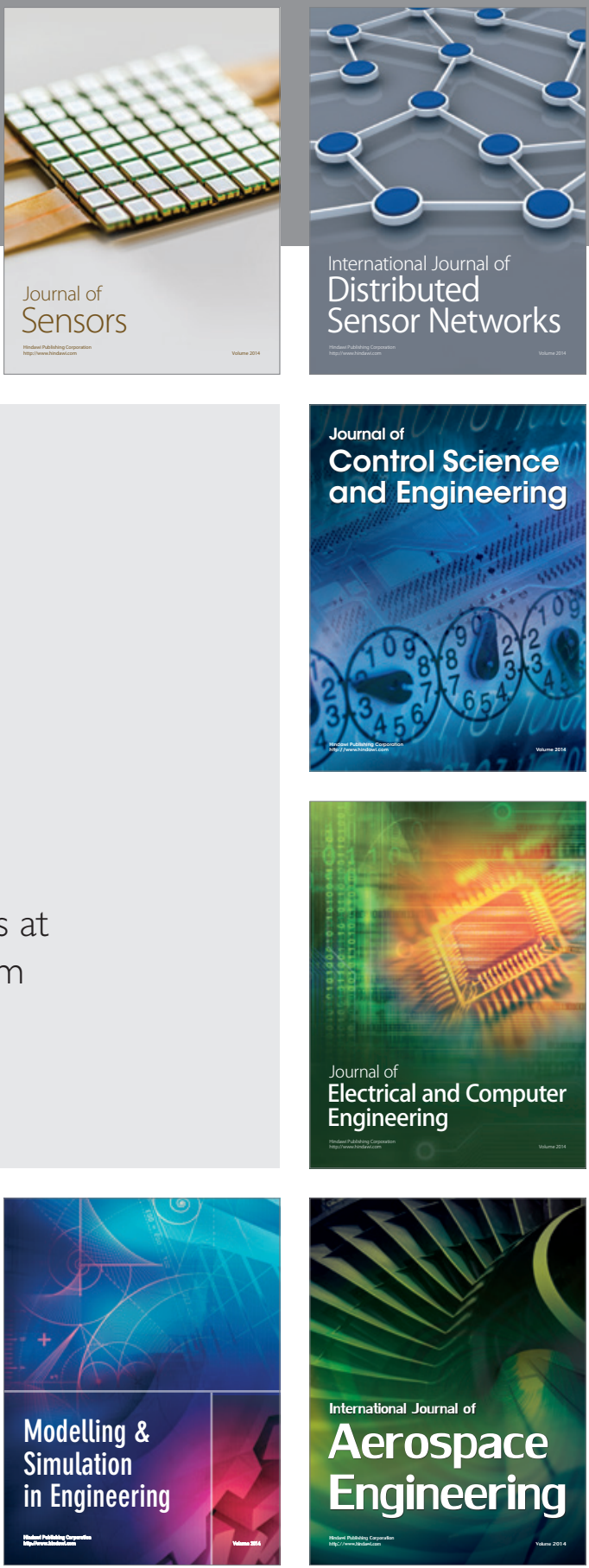

Journal of

Control Science

and Engineering
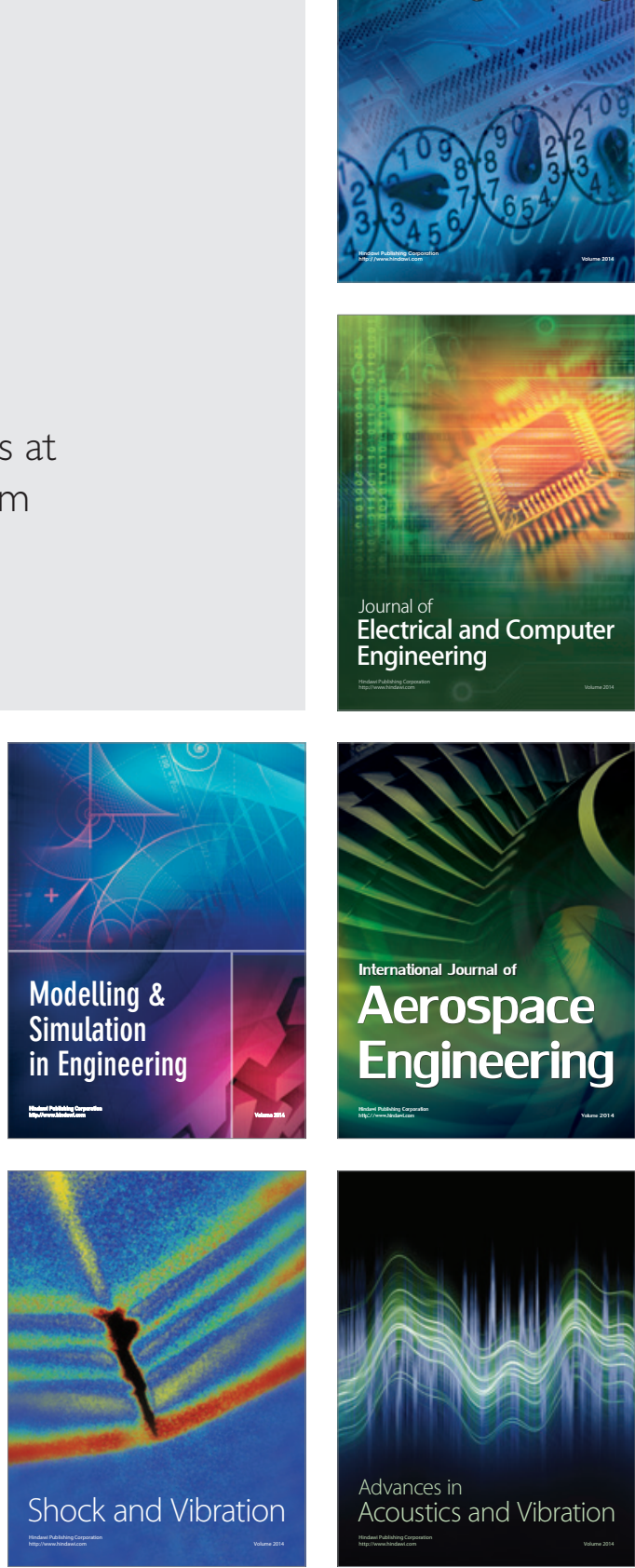